\title{
Novel prognostic biomarkers of gastric cancer based on gene expression microarray: COL12A1, GSTA3, FGA and FGG
}

\author{
SHIJIE DUAN ${ }^{*}$, BAOCHENG GONG ${ }^{*}$, PENGLIANG WANG, HANWEI HUANG, LEI LUO and FUNAN LIU \\ Department of Surgical Oncology, The First Affiliated Hospital of China Medical University, \\ Shenyang, Liaoning 110001, P.R. China
}

Received February 8, 2018; Accepted August 2, 2018

DOI: $10.3892 / \mathrm{mmr} .2018 .9368$

\begin{abstract}
Gastric cancer (GC) is the fifth most common malignancy and the third leading cause of cancer-associated mortality in the world. However, its mechanisms of occurrence and development have not been clearly elucidated. Furthermore, there is noeffective tumor marker for GC.Using DNA microarray analysis, the present study revealed genetic alterations, screened out core genes as novel markers and discovered pathways for potential therapeutic targets. Differentially expressed genes (DEGs) between GC and adjacent normal tissues were identified, followed by pathway enrichment analysis of DEGs. Next, the protein-protein interaction (PPI) network of DEGs was built and visualized. Analyses of modules in the PPI network were then performed to identify the functional core genes. Finally, survival analysis of core genes was conducted. A total of 256 genes were identified as DEGs between the GC samples and normal samples, including 169 downregulated and 87 upregulated genes. Through Gene Ontology (GO) and Kyoto Encyclopedia of Genes and Genomes pathway enrichment analysis, the present study identified a total of 143 GO terms and 21 pathways. Six clusters of functional modules were identified, and the genes associated with these modules were
\end{abstract}

Correspondence to: Professor Funan Liu, Department of Surgical Oncology, The First Affiliated Hospital of China Medical University, 155 Nanjing North Street, Heping, Shenyang, Liaoning 110001, P.R. China

E-mail:1fn540@126.com

*Contributed equally

Abbreviations: GC, gastric cancer; DEGs, differentially expressed genes; GO, Gene Ontology; KEGG, Kyoto Encyclopedia of Genes and Genomes; PPI, protein-protein interaction; CEA, carcinoembryonic antigen; CA 19-9, carbohydrate antigen 19-9; CA-50, carbohydrate antigen 50; CHGA, Chromogranin A; THY1, Thy-1 cell surface antigen; BP, biological process; CC, cellular component; MF, molecular function; GST, glutathione S-transferases; KM, Kaplan-Meier

Key words: gastric cancer, biomarker, differentially expressed genes, PPI network, modules, KM plotter screened out as the functional core genes. Certain core genes, including collagen type $12 \alpha 1$ chain (COL12Al), glutathione S-transferase $\alpha 3$ (GSTA3), fibrinogen $\alpha$ chain $(F G A)$ and fibrinogen $\gamma$ chain $(F G G)$, were the first reported to be associated with GC. Survival analysis suggested that these four genes, COL12A1 ( $\mathrm{P}=0.002)$, GSTA3 $\left(\mathrm{P}=3.4 \times 10^{-6}\right), F G A(\mathrm{P}=0.00075)$ and $F G G\left(\mathrm{P}=1.4 \times 10^{-5}\right)$, were significant poor prognostic factors and therefore, potential targets to improve diagnosis, optimize chemotherapy and predict prognostic outcomes.

\section{Introduction}

Gastric cancer (GC) is the fifth most common malignancy in the world, especially in developing countries (1). GC has been the leading cause of cancer death worldwide until the mid-1990s, after which the occurrence has been substantially declining. However, certain countries in Eastern Asia, such as China, Korea and Japan, remain as highly endemic areas (2). In China, the 5-year survival rate of GC has improved $16 \%$ for patients diagnosed during 1995-2009 (3). However, GC is still the third leading cause of cancer death in the world and its prognosis is relatively poor (1).

In recent years, a growing number of researchers devoted to $\mathrm{GC}$ research and achieved considerable achievements. In the previous studies, various molecular mechanisms and biomarkers related to GC have been identified. Through a retrospective study, Pectasides et al (4) found that using carcinoembryonic antigen (CEA), carbohydrate antigen 19-9 (CA 19-9) and carbohydrate antigen 50 (CA-50) as GC biomarkers had significant value in early detection of recurrence and monitoring progression. Ara et al (5), discovered that non-canonical Wnt signaling pathway contributed to GC progression, which may serve as a new potential therapeutic target for GC. Despite of these advances, the mechanisms of GC occurrence and development are yet to be clearly elaborated. Furthermore, the biomarkers commonly used for GC diagnosis and treatment, including carbohydrate antigen 125 (CA125), CA19-9 and CEA, have significant sensitivity and specificity issues (6). As such, further research on revealing genetic alterations, identification of new biomarkers and exploration of pathways associated with GC is critically needed.

DNA microarray analysis is a rather powerful method to screen out cancer-related genes as novel diagnostic and prognostic markers and disclose genetic alterations of cancer 
evolution and progression. For example, Yang et al (7) found that Chromogranin A (CHGA) and Thy-1 cell surface antigen (THY1) were novel biomarkers in the diagnosis of cancer via DNA microarray analysis. Similarly, Sun et al (8) identified the COL family as promising prognostic markers for GC. Often used in combination with DNA microarray analysis, gene ontology (GO) enrichment analysis is a strategy to characterize the function categories affected by cancer and Kyoto Encyclopedia of Genes and Genomes (KEGG) pathway enrichment analysis is an approach used to find out biologic pathways enriched in cancer. For example, with the above analysis methods, Hu et al (9) confirmed that several pathways, including focal adhesion, ECM-receptor interactions and the metabolism of xenobiotics by cytochrome P450, were associated with the progression of GC. Another important technique, modules analysis, is more crucial to study the specific behavior of modules and identify functional genes as cancer biomarkers compared with genes with straightforward high interaction degree (10).

In the present study, we selected expression profile (GSE79973) to perform DNA microarray analysis to reveal genetic alterations, identify new biomarkers and explore novel pathways associated with GC. In addition to biomarkers previously reported, we identified four novel biomarkers, COL12A1, GSTA3, FGA, and FGG, as significant poor prognostic factors. Most of the enriched GO terms and KEGG pathways of those genes are related to GC, such as chemical carcinogenesis, metabolism of xenobiotics by cytochrome P450, ECM-receptor interaction, focal adhesion, and platelet activation. These findings may provide insights on GC occurrence and development, as well as potential therapeutic targets for future research.

\section{Materials and methods}

Microarray data. To account for tumor heterogeneity, we conducted DNA microarray analysis with the new gene profiles, GSE79973, to identify genes as novel biomarkers. In the present study, the expression profiles associated with GC, GSE79973, were downloaded from GEO (www.ncbi.nlm.nih. gov/geo/), which is a public functional genomic data repository. GSE79973 containing 20 tissues (10 pairs of GC tissues and adjacent non-tumor tissues) were obtained from Zhejiang Provincial People's Hospital, Zhejiang, China. The platform was based on GPL570 [HG-U133_Plus_2] Affymetrix Human Genome U133 Plus 2.0.

Preprocess microarray data. The Microarray Data was preprocessed by affy package in R (11). The purpose of this step was to filter out unwanted noise of the raw microarray and ensure background and data were standardized (12). Furthermore, for these genes that corresponded with multiple probes, we used the average expression values of those probes as the expression value of each gene. Furthermore, to visualize the difference before and after normalization, we constructed box plots of raw and normalization data.

Identification of the DEGs. Using LIMMA package in R, we collected a data list from GSE79973 (13). We then screened out these DEGs by means of $\mathrm{t}$-test with P-value $<0.05$ and
$\| \log 2$ (fold change) $\mid>2$. The heatmap and volcano map of these DEGs were constructed using pheatmap package in $\mathrm{R}$ and gplots package in $\mathrm{R}$, respectively.

GO terms and KEGG pathways of DEGs. To elaborate how DEGs affected the GC cells, GO and KEGG pathway enrichment analyses of DEGs were conducted using DAVID (david. abcc.ncifcrf.gov/). The DAVID database includes a total of four modules (functional annotation, gene functional classification, gene ID conversion and gene name batch viewer). The database for annotation was used in the presnt study. The GO terms and pathways of DEGs with $\mathrm{P}<0.05$ and at least five genes were screened out as significant function annotation of DEGs.

PPI network analysis of the DEGs. To study the molecular interactions between DEGs, we built the PPI network using the STRING (www.string-db.org/) database (14). The PPI network includes direct (physical) and indirect (functional) associations and stem from computational prediction, knowledge transfer between organisms, and interactions aggregated from other (primary) databases. Using the STRING database, we could obtain certain integrated scores of interactions among DEGs and select out the genes whose integrated scores were bigger than 0.4 (the default threshold in the STRING database). The PPI networks were then visualized by the Cytoscape software (15).

Sub-network modeling analysis of PPI networks. In PPI networks, genes in the same module typically show the same or similar function and work together to implement their biological function. To visualize the network and identify the modules in the network, MCODE plug-in on the Cytospace software (www.cytoscape.org/) was used. The parameters were set as follows: Degree cutoff $\geq 2$ (degrees of each node in module were at least larger than 2), K-core $\geq 2$ (subgraphs of each node in module were at least more than 2). After that, the GO enrichment analysis was performed using DAVID and the functional core genes of subsets were selected in each module.

Survival analysis of core genes. The survival analysis of core genes was conducted by Kaplan-Meier plotter (KM plotter, www.kmplot.com), whose gene expression data as well as relapse free and overall survival information are downloaded from the well-known public database, including GEO (Affymetrix microarrays only), EGA and TCGA. The Kaplan Meier (KM) plotter is capable of assessing the effect of 54,675 genes on survival using 10,461 cancer samples, 1,065 of which are GC patients with a mean follow-up of 69/40/49/33 months. Based on the KM plotter on the webpage, the hazard ratio (HR) with $95 \%$ confidence intervals and log rank P-values were calculated and the curves were generated.

\section{Results}

Data preprocessing. Genes with systematic bias among original data were removed after preprocessing using the Affy package in $\mathrm{R}$ software. The expression data of genes before and after normalization are shown in Fig. 1. The black lines in 

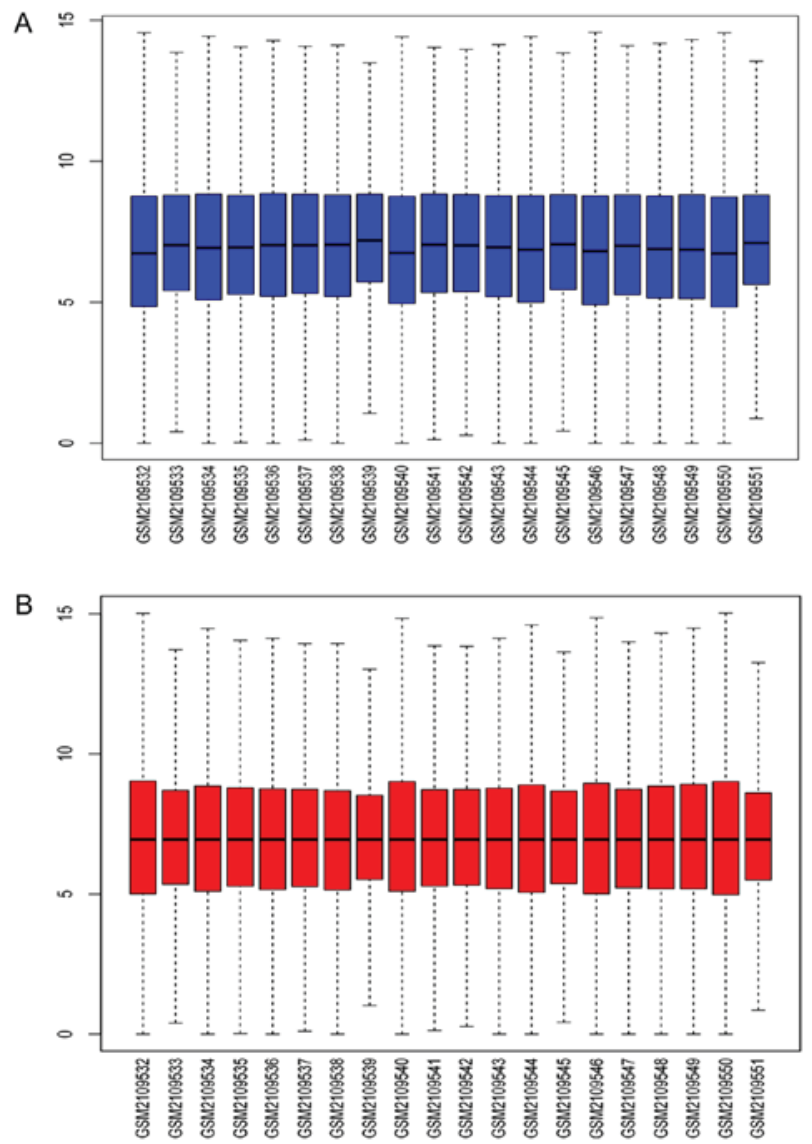

Figure 1. Normalized expression value data. The black line in each box represents the median of each set of data, which determined the degree of standardization of data through its distribution. (A) Box plots of expressed value data prior to normalization. (B) Box plots of expressed value data following normalization. The gene expression value is presented on the $\mathrm{y}$-axis and the samples on the $\mathrm{x}$-axis.

each of the boxes represent the medians of each dataset. The black lines are shown at almost the same level in the box plots, indicating a significant effect of standardization.

Get the DEGs. Using the R package LIMMA, we identified the DEGs between the GC and normal samples. A total of 256 DEGs in the data list were identified from GSE79973, including 169 down-regulated and 87 up-regulated genes. We ranked these DEGs by P-value and constructed the heatmap (Fig. 2) as well as volcano map (Fig. 3). Based on the heatmap and volcano map, the gene expressions of these DEGs exhibit significant differences.

GO term enrichment analysis of the DEGs. Using the DAVID and pathway enrichment analysis of the DEGs, we identified a total of $100 \mathrm{GO}$ terms and 16 pathways. The top 10 enriched GO terms of the DEGs based on the P-values are listed (Table I). As shown in Table I, DEGs were significantly enriched in biological processes (BP), including extracellular matrix organization, skeletal system development, cell adhesion and xenobiotic metabolic process. For molecular function (MF), DEGs were significantly enriched in extracellular matrix structural constituent and extracellular matrix binding. In addition, $\mathrm{GO}$ cell component $(\mathrm{CC})$ analysis indicated that the DEGs were significantly enriched in extracellular space,

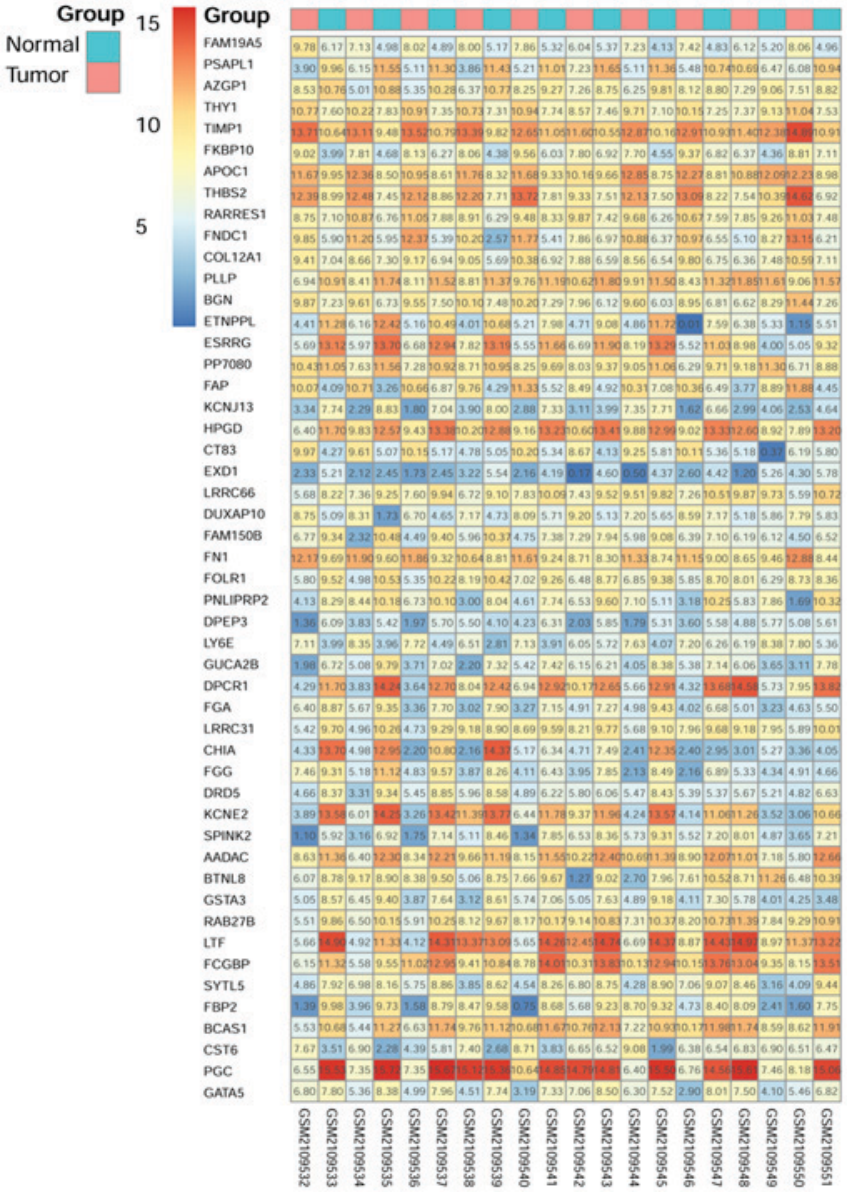

Figure 2. Heatmap of the DEGs. Overview of the associations between DEGs from normal and GC tissues. Each row represents the tissues (10 GC and 10 normal). Red coloration indicated $10 \mathrm{GC}$ tissues. Blue coloration indicated 10 adjacent normal tissues. Each column represents the gene IDs. Log ratio scale bar for the Treeview color change was also presented. The red in the log ratio scale bar indicates highly expressed genes in GC tissues when compared with the normal tissues, while the blue represents the low expression of genes. The number in each block with its color in the log ratio scale bar represents the expression level of each gene in different samples. GC, gastric cancer; DEGs, differentially expressed genes.

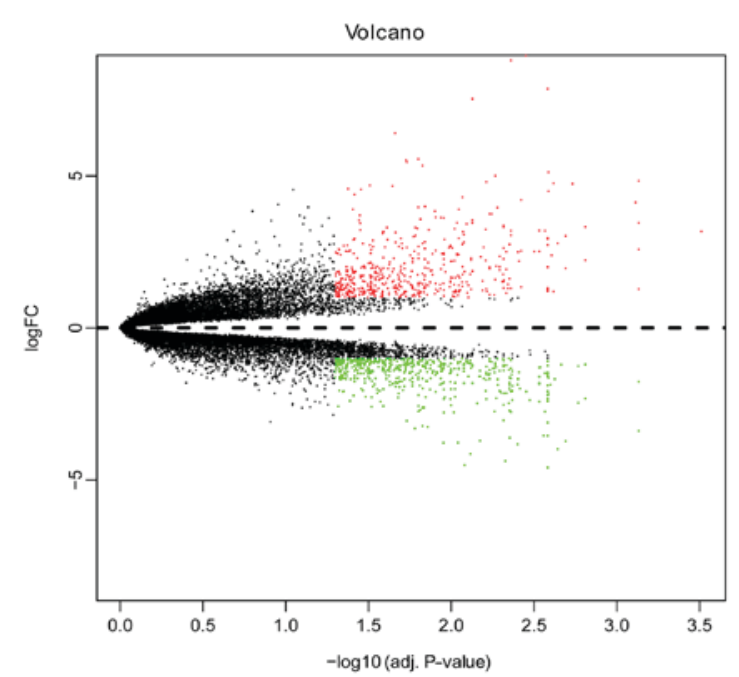

Figure 3. Volcano plot overview of the distribution of DEGs. Red spots indicate upregulated genes, while green spots indicate downregulated genes. Black spots represent genes that were not DEGs. The-log10 (adj. P-value) on the $\mathrm{x}$-axis is the $\mathrm{P}$-value following correction and the $\log 2 \mathrm{FC}$ value on the $\mathrm{y}$-axis represents the fold change of genes. DEGs, differentially expressed genes. 
Table I. Top 10 enriched GO terms, which were sorted by P-value in ascending order.

\begin{tabular}{|c|c|c|c|c|}
\hline Category & GO ID & GO name & P-value & Gene number \\
\hline $\mathrm{CC}$ & GO:0005615 & Extracellular space & $1.07 \times 10^{-15}$ & 55 \\
\hline $\mathrm{CC}$ & GO:0005576 & Extracellular region & $4.88 \times 10^{-15}$ & 59 \\
\hline $\mathrm{BP}$ & GO:0030198 & Extracellular matrix organization & $1.99 \times 10^{-11}$ & 19 \\
\hline $\mathrm{CC}$ & GO:0005578 & Proteinaceous extracellular matrix & $3.87 \times 10^{-9}$ & 19 \\
\hline $\mathrm{CC}$ & GO:0005581 & Collagen trimer & $1.12 \times 10^{-8}$ & 12 \\
\hline BP & GO:0001501 & Skeletal system development & $9.95 \times 10^{-9}$ & 14 \\
\hline BP & GO:0007155 & Cell adhesion & $2.95 \times 10^{-8}$ & 23 \\
\hline MF & GO:0005201 & Extracellular matrix structural constituent & $9.78 \times 10^{-7}$ & 9 \\
\hline $\mathrm{BP}$ & GO:0006805 & Xenobiotic metabolic process & $3.84 \times 10^{-5}$ & 8 \\
\hline $\mathrm{MF}$ & GO:0050840 & Extracellular matrix binding & $2.09 \times 10^{-4}$ & 5 \\
\hline
\end{tabular}

GO, Gene Ontology; BP, biological process; CC, cellular component; MF, molecular function.

Table II. KEGG pathways enriched in DEGs.

\begin{tabular}{|c|c|c|c|}
\hline Category & Pathway name & Gene number & P-value \\
\hline KEGG_PATHWAY & hsa05204:Chemical carcinogenesis & 11 & $1.65 \times 10^{-7}$ \\
\hline KEGG_PATHWAY & hsa04974:Protein digestion and absorption & 11 & $4.12 \times 10^{-7}$ \\
\hline KEGG_PATHWAY & hsa00980:Metabolism of xenobiotics by cytochrome P450 & 10 & $9.02 \times 10^{-7}$ \\
\hline KEGG_PATHWAY & hsa04512:ECM-receptor interaction & 10 & $3.59 \times 10^{-6}$ \\
\hline KEGG_PATHWAY & hsa00982:Drug metabolism-cytochrome P450 & 9 & $4.86 \times 10^{-6}$ \\
\hline KEGG_PATHWAY & hsa00830:Retinol metabolism & 7 & $3.10 \times 10^{-4}$ \\
\hline KEGG_PATHWAY & hsa04971:Gastric acid secretion & 7 & $5.83 \times 10^{-4}$ \\
\hline KEGG_PATHWAY & hsa00010:Glycolysis/Gluconeogenesis & 6 & 0.0027 \\
\hline KEGG_PATHWAY & hsa04510:Focal adhesion & 10 & 0.0027 \\
\hline KEGG_PATHWAY & hsa04978:Mineral absorption & 5 & 0.0041 \\
\hline KEGG_PATHWAY & hsa00350:Tyrosine metabolism & 4 & 0.0134 \\
\hline KEGG_PATHWAY & hsa05146:Amoebiasis & 6 & 0.0177 \\
\hline KEGG_PATHWAY & hsa00071:Fatty acid degradation & 4 & 0.0247 \\
\hline KEGG_PATHWAY & hsa01100:Metabolic pathways & 26 & 0.0379 \\
\hline KEGG_PATHWAY & hsa04611:Platelet activation & 6 & 0.0383 \\
\hline KEGG_PATHWAY & hsa04972:Pancreatic secretion & 5 & 0.0440 \\
\hline
\end{tabular}

DEGs, differentially expressed genes; KEGG, Kyoto Encyclopedia of Genes and Genomes.

extracellular region, proteinaceous extracellular matrix and collagen trimer.

KEGG pathway analysis of the DEGs. A total of 16 enriched pathways with $\mathrm{P}<0.05$ were shown in Table II. The first enriched pathway, chemical carcinogenesis, was directly related to cancer and all the others have been reported to play an important role in cancer progression via certain biological processes, such as protein digestion and absorption, metabolism of xenobiotics by cytochrome P450, ECM-receptor interaction, focal adhesion, metabolic pathways, and platelet activation.

PPI network of DEGs. The whole PPI network shown in Fig. 4 contained 116 nodes and 197 edges. The nodes represent DEGs with higher integrated scores of interactions and the edges represent interactions among the DEGs. The genes with interaction degree $\geq 5$ were shown in Table III. COL1A2, COL2A1, COL11A1 and SPARC, whose corresponding degree was beyond 10 , were hub genes in the PPI network and closely related to cancer.

Modules in PPI network. Six modules were identified in this study, including 8, 6, 3, 3, 3 and 3 genes, respectively (Fig. 5). The top three GO terms of each modules are shown in Table IV. Interestingly, the GO terms of these modules were mainly associated with collagen trimer, (S)-limonene 7-monooxygenase activity, extracellular matrix organization, multicellular organism development, glutathione derivative biosynthetic process and fibrinogen complex, respectively. Among the core genes involved in these modules, COL12A1, 


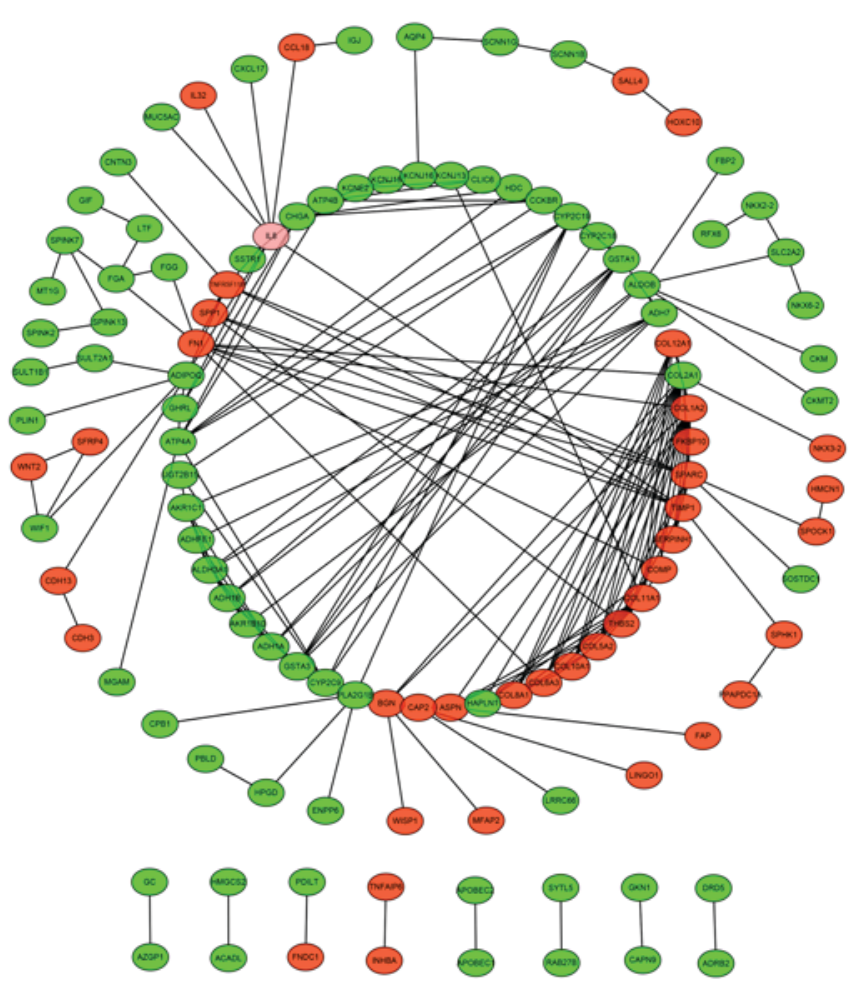

Figure 4. PPI network of DEGs. The network contained 116 nodes and 197 edges. The 39 red nodes were the upregulated genes and the 76 green nodes were the downregulated genes. The one pink node indicates a gene that was automatically generated and was potential associated with DEGs. The edges represented the correlation between two genes. PPI, protein-protein interaction; DEGs, differentially expressed genes.

GSTA3, FGA and $F G G$ were firstly reported to be associated with GC.

Kaplan-Meier survival curves. The Kaplan-Meier (KM) survival curves are shown in Fig. 6. Using the KM plotter platform, the GC patients were divided into two groups, with low and high expression of the four core genes, respectively. We found that each of the four core genes, COL12A1 [HR 1.4 (1.13-1.74) $\mathrm{P}=0.002$ ], GSTA3 [HR 1.64 (1.33-2.02) $\mathrm{P}=3.4 \mathrm{e}-06$ ], FGA [HR 1.36 (1.14-1.63) $\mathrm{P}=0.00075]$ and $F G G$ [HR 1.45 (1.23-1.72) $\mathrm{P}=1.4 \mathrm{e}-05]$, was a poor prognostic factor of overall survival.

\section{Discussion}

$\mathrm{GC}$, as one of the most malignant tumor, was a typical heterogeneous cancer with molecular complexity and heterogeneity (16,17). Recently, a substantial number of effort have been paid in exploring the underwent molecular mechanism of GC and several genetic and epigenetic alterations have been identified as biomarkers with effect on diagnosis, treatment, stratification and prognosis (18-20). However, with the technological improvement, its heterogeneity was found to be more complex than previous imagined, regarding genomic instability, differentially expressed genes (DEGs), genetic variations, epigenetic heterogeneity, protein heterogeneity and so on $(21,22)$. The heterogeneity of GC presented some complex biological characteristics, such as recurrence and metastasis of cancer, sensitivity to adjuvant therapy, and so on (23-25). Therefore, these identified biomarkers still were
Table III. Core genes with corresponding degree $\geq 5$.

\begin{tabular}{|c|c|}
\hline Gene & Degree \\
\hline COL1A2 & 15 \\
\hline COL2A1 & 15 \\
\hline COL11A1 & 12 \\
\hline SPARC & 11 \\
\hline COL5A2 & 9 \\
\hline FN1 & 9 \\
\hline IL8 & 9 \\
\hline TIMP1 & 9 \\
\hline ALDH3A1 & 8 \\
\hline ATP4A & 8 \\
\hline COL6A3 & 8 \\
\hline GSTA1 & 8 \\
\hline GSTA3 & 8 \\
\hline THBS2 & 8 \\
\hline ADH1A & 7 \\
\hline ADH1B & 7 \\
\hline ADH7 & 7 \\
\hline ADIPOQ & 7 \\
\hline ALDOB & 7 \\
\hline COL10A1 & 7 \\
\hline COL12A1 & 7 \\
\hline COL8A1 & 7 \\
\hline TNFRSF11B & 7 \\
\hline BGN & 6 \\
\hline CYP2C19 & 6 \\
\hline CYP2C9 & 6 \\
\hline PLA2G1B & 6 \\
\hline AKR1C1 & 5 \\
\hline ASPN & 5 \\
\hline CHGA & 5 \\
\hline COMP & 5 \\
\hline
\end{tabular}

ineffective and inconstant, which slow their clinical application $(17,26)$. Even so, it still should not be neglected that exploring novel biomarkers was helpful to further construct the molecular network of GC, improve our understanding on heterogeneity, and identify more meaningful genetic subtypes related to individual characteristics, such as prognosis, sensitivity of adjuvant therapy and so on (21).

In our study, a total of six modules were identified. These modules were analyzed with these DEGs that met with the default parameters. And the expression data of these DEGs were identified based on the gene profiles (GSE79973). Therefore, as a result of the tumor heterogeneity, these modules were associated with this gene profiles. Interestingly, from the function annotation of these modules, on the one hand, these modules were enriched in some common cancer signaling pathway such as PI3K-Akt signaling pathway, Wnt signaling pathway and so on $(27,28)$. On the other hand, these modules also were enriched in the processes, such as gastric acid secretion, protein digestion and absorption and so on, which were specific in the GC. 

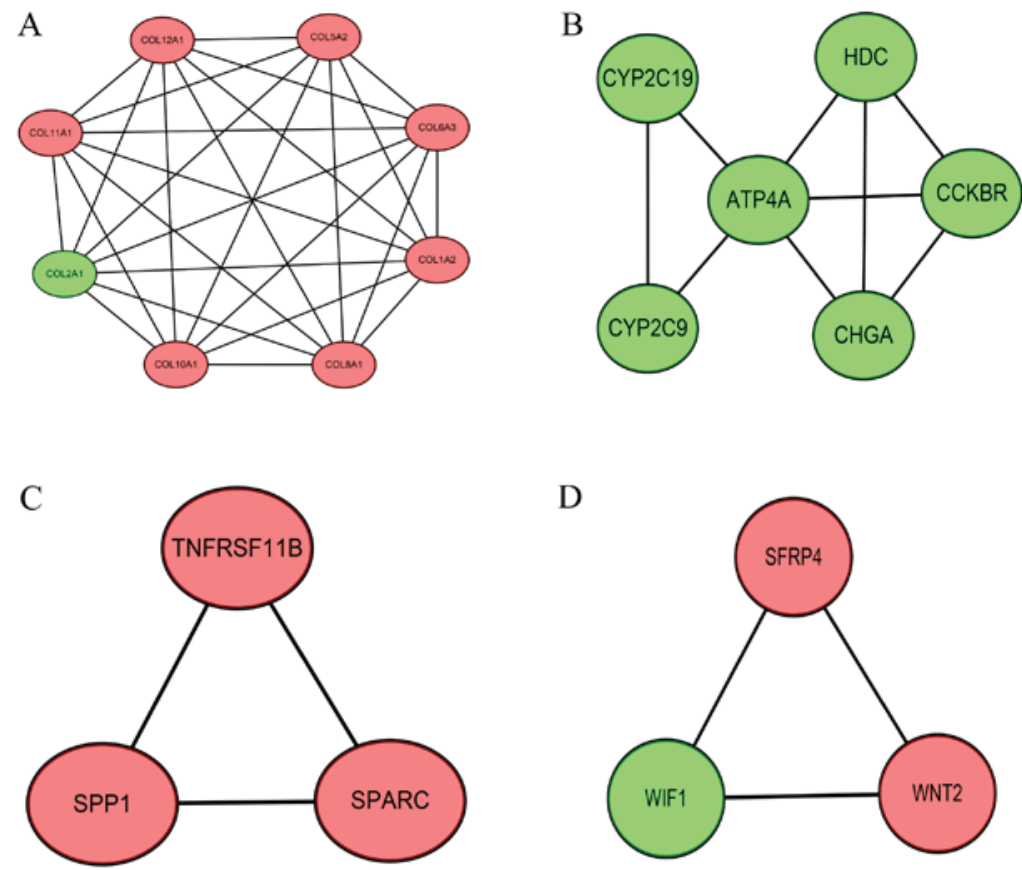

D
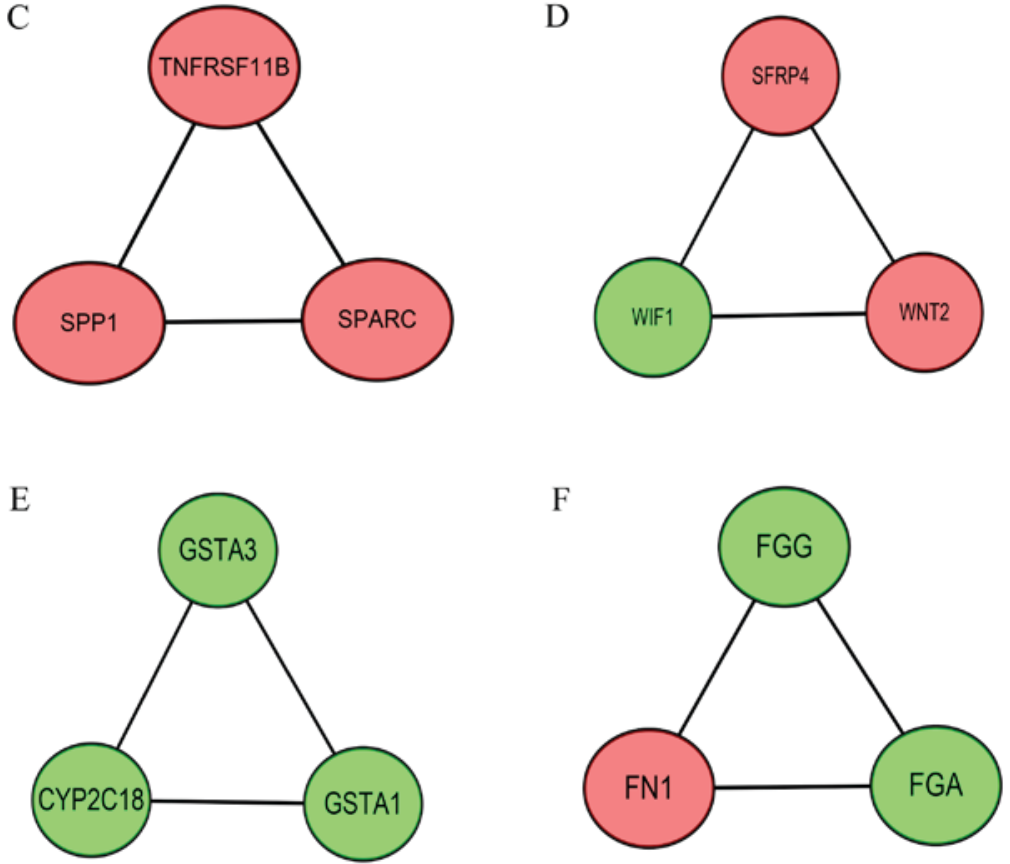

Figure 5. Functional modules involved in the PPI network. The red nodes represent the upregulated genes and the green nodes represent the downregulated genes. The edges indicate the correlation between two genes. (A) Module A was comprised of 8 nodes and 28 edges. (B) Module B was comprised of 6 nodes and 9 edges. (C) Module $\mathrm{C}$ was comprised of 3 nodes and 3 edges. (D) Module D was comprised of 3 nodes and 3 edges. (E) Module E was comprised of 3 nodes and 3 edges. (F) Module F was comprised of 3 nodes and 3 edges. PPI, protein-protein interaction.

We also found that certain well-known gastric prognosis factors, such as some non-canonical Wnt signaling genes and PICT1, were not included in the identified modules (29). In fact, these well-known gastric prognosis factors were identified as DEGs in our study. But they were not included in further module analysis for their expression level without meeting with the default parameter. This issue resulted from the tumor heterogeneity. It is the heterogeneity that restricts these well-known prognostic factors as sites of targeted therapy, for example trastuzumab for the GC patients with HER-2 positive (30). The trastuzumab has been a standard treatment strategy for the GC patients with HER-2 positive (31). However, only a small number of patients can benefit from it, which mainly results from HER-2 in a fraction of GC patients ( 7-34\%) being overexpression due to the heterogeneity (32). Even so, the tumor heterogeneity makes it possible to explore some novel genes related to prognosis of patients with GC as the candidates of biomarkers. These novel genes may not be as important as these well-known prognostic genes. But It is helpful to construct tumor molecular network and provide more accurate individualized treatment plan. Remarkably, we identified four GC-associated functional core genes, COL12A1 in Module A, GSTA3 in Module E and FGA, FGG in Module $\mathrm{F}$, and the level of these genes correlate with poor prognosis.

COL12Al was reported to be related to several types of cancers, such as subungual exostosis, ovarian, breast and colon cancer, which suggest that COL12Al may be a new potential cancer biomarker (33-36). As a member of the FACIT (fibril associated collagens with interrupted triple helices) collagen family, COL12A1, together with COL6A3, COL8A1, COL1A2, COL5A2, COL10A1, COL11A1 and COL2A1, has the molecular function of extracellular matrix structural constituent. These FACIT members take part in the biological processes of collagen fibril organization and construct the cellular component of collagen trimer. Although COL12A is expressed in a variety of tumor tissues, its exact function remains poorly understood. Januchowski et al (34) found that COL12AI played a role in the drug resistance of cancer cells and tumor progression. Based on our observed GO terms and pathways in module A (Fig. 5), COL12Al appear to participate in pathways of protein digestion and absorption as well as focal adsorption, which may allow tumor cells to invade into the surrounding 
Table IV. Top three GO terms in each module.

\begin{tabular}{|c|c|c|c|}
\hline GOID & GO name & P-value & Genes ID \\
\hline \multicolumn{4}{|l|}{ Module A } \\
\hline 0005581 & Collagen trimer & $2.49 \times 10^{-9}$ & $\begin{array}{l}\text { COL6A3, COL1A2, COL2A1, COL8A1, } \\
\text { COL10A1 }\end{array}$ \\
\hline 0030199 & Collagen fibril organization & $1.17 \times 10^{-7}$ & COL1A2,COL2A1,COL5A2,COL11A1 \\
\hline 0005201 & Extracellular matrix structural constituent & $1.19 \times 10^{-7}$ & COL1A2,COL2A1,COL5A2,COL11A1 \\
\hline \multicolumn{4}{|l|}{ Module B } \\
\hline 0018676 & (S)-limonene 7-monooxygenase activity & $4.74 \times 10^{-4}$ & CYP2C19, CYP2C9 \\
\hline 0018675 & Limonene 6-monooxygenase activity & $4.74 \times 10^{-4}$ & CYP2C19, CYP2C9 \\
\hline 0052741 & (R)-limonene 6-monooxygenase activity & $4.74 \times 10^{-4}$ & CYP2C19, CYP2C9 \\
\hline \multicolumn{4}{|l|}{ Module C } \\
\hline 0030198 & Extracellular matrix organization & $1.36 \times 10^{-4}$ & TNFRSF11B, SPARC, SPP1 \\
\hline 0050840 & Extracellular matrix binding & 0.0031 & SPARC, SPP1 \\
\hline 0005615 & Extracellular space & 0.0055 & TNFRSF11B, SPARC, SPP1 \\
\hline \multicolumn{4}{|l|}{ Module D } \\
\hline 0007275 & Multicellular organism development & $9.61 \times 10^{-4}$ & WNT2, SFRP4, WIF1 \\
\hline 0017147 & Wnt-protein binding & 0.0037 & SFRP4, WIF1 \\
\hline 0030178 & Negative regulation of Wnt signaling pathway & 0.0062 & SFRP4, WIF1 \\
\hline \multicolumn{4}{|l|}{ Module E } \\
\hline 1901687 & Glutathione derivative biosynthetic process & 0.0026 & GSTA1, GSTA3 \\
\hline 0004364 & Glutathione transferase activity & 0.0041 & GSTA1, GSTA3 \\
\hline 0006749 & Glutathione metabolic process & 0.0067 & GSTA1, GSTA3 \\
\hline \multicolumn{4}{|l|}{ Module F } \\
\hline 0005577 & Fibrinogen complex & $2.17 \times 10^{-7}$ & FGG, FGA, FN1 \\
\hline 0031093 & Platelet $\alpha$ granule lumen & $8.94 \times 10^{-6}$ & FGG, FGA, FN1 \\
\hline 0002576 & Platelet degranulation & $3.73 \times 10^{-5}$ & FGG, FGA, FN1 \\
\hline
\end{tabular}

GO, Gene Ontology.

microenvironment. In addition, high expression of COL12AI is associated with poor prognosis. Thus, $C O L 12 \mathrm{Al}$ may be a new potential marker and prognostic factor of $\mathrm{GC}$ and its pathways may be potential treatment targets.

GSTA3 was identified to be a down-regulated gene in our study. Together with GSTA1 and CYP2C18, GSTA3 constructed the Module E (Fig. 5). They interact with each other and participate in chemical carcinogenesis, glutathione metabolism and drug metabolism-cytochrome P450. GSTA3 and GSTA1 encode a superfamily of glutathione S-transferases (GST), which are involved in biotransformation of toxic xenobiotics and endobiotics, as well as arachidonic acid metabolism via conjugation with reduced glutathione (GSH) (37). The expression level of GST are associated with the resistance of cells to toxic chemicals, such as carcinogens, antitumor drugs, environmental pollutants, and products of oxidative stress (38). Therefore, GSTA3 may be a potential marker in selecting chemotherapy drug (39). Further research on the association of these molecules is needed to help us better understand the exact role of GSTA3 in GC regulation.

$F G A$ and $F G G$ encode the alpha and gamma components of fibrinogen, which are constituent parts of blood-borne glycoprotein (40). They were reported to differentially express in many types of cancer, such as prostate cancer, lung cancer, hepatocellular carcinoma, and pancreatic cancer (40-43). Plasma fibrinogen levels before surgery together with histological grade and lymph node involvement have been defined as independent prognostic factors in patients with colorectal cancer (44). Ghezzi et al (45), reported that plasma fibrinogen level might be a potential index to predict prognosis, improve the early diagnosis of endometrial cancer and optimize the treatment schedule. The possible mechanisms behind the association of plasma fibrinogen level with cancer include (1) the soluble form of fibrinogen may serve as the bridging molecule between tumor cells and host cells; (2) tumor cells and platelets may form large aggregates, which prevent tumor cells from being attacked by the innate immune system and (3) fibrinogen is essential to the sustained adherence of tumor cells to the endothelia of target tissues (46-48). In $\mathrm{GC}$, fibrinogen plays a key role in hematogenous and lymphatic metastasis of cancer cells through spontaneous metastasis, facilitating the stable adhesion and/or survival of metastatic emboli after tumor cell intravasation (49). The plasma fibrinogen level on the prognosis was based on staging of tumor with worse prognosis in T2 GC as well 

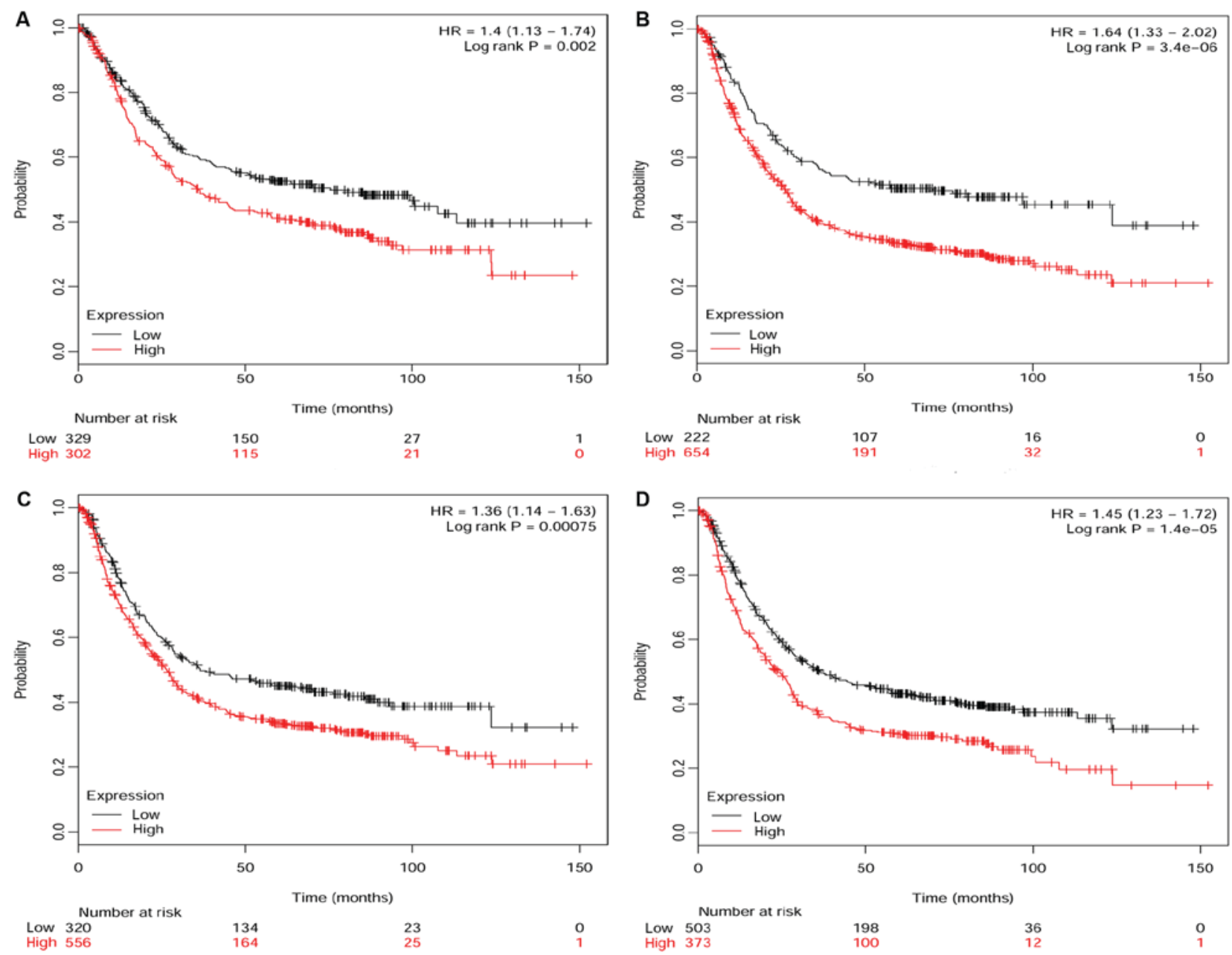

Figure 6. Kaplan-Meier survival curves of four genes generated from Kaplan-Meier plotter. Curves of (A) COL12A1, (B) GSTA3, (C) FGA and (D) FGG. COL12A1, collagen type $12 \alpha 1$ chain; GSTA3, glutathione S-transferase $\alpha 3 ; F G A$, fibrinogen $\alpha$ chain; FGG, fibrinogen $\gamma$ chain; HR, hazard ratio; CI, confidence interval.

as lymphatic and hematogenous metastasis, however, not in T3/T4 GC (50). However, they were directly reported to be associated with GC for the first time in this study. There still existed debate in expression level about these genes in tissues of cancer (51-53). In our study, FGA and $F G G$ were identified to be down-regulated genes, while their high expression indicated poor prognosis for patients with GC. Besides tumor, many other factors may influence the alteration of expression level of $F G G$ and $F G A$, such as acute-phase reactant and binding sites in tissues $(40,54)$. Furthermore, levels of fibrinogen genes ( $F G A$ and $F G G$ ) changed following a circadian rhythms pattern and this additional level of fibrinogen transcriptional regulation has not yet been characterized (55). Considering pulmonary infarction as one of the most serious complications after GC surgery and the relationships between plasma fibrinogen level and GC, we should take it into consideration whether postoperative patients need a routine prophylactic anticoagulation. As evaluation indexes of blood coagulation state, $F G G$ and $F A G$ are potential biomarkers to improve diagnosis, optimize treatment and predict prognosis.

We have a lot of limitations in the universality and applicability of these novel biomarkers for the observational nature of this article. Our study was conducted based on the gene profiles, GSE79973, which contained 10 pairs of GC tissues and adjacent non-tumor tissues. The association of the identified genes and GC, their value to predict prognosis and molecular interactions among these genes were not verified in clinical samples by experiments. For above reasons, the further experiments and studies are needed to measure the identified genes in clinical practice and investigate the biological mechanisms of the interactions among these identified genes.

In conclusion, COL12A1, GSTA3, FGA and $F G G$ have been identified to be associated with GC in this study and were selected as the core genes. Through GO and pathway enrichment of modules, we identified the functions and pathways of these core genes. Furthermore, based on survival analysis, the four genes were found to be significant poor prognostic factors. These core genes might be potential markers to improve diagnosis, optimize chemotherapy plan and predict prognosis. In addition, the pathways related to these genes may be potential therapeutic targets for GC. We plan to embark on validating the potential functions and pathways of these genes in our future studies.

\section{Acknowledgements}

Not applicable. 


\section{Funding}

The present study was supported by the National Natural Science Foundation of China (grant no. 81001092), CSCO-Merck Serono Cancer Research Fund (grant no. Y-MX2016-013) and the Natural Science Foundation of Liaoning Province of China (grant no. 2013021097).

\section{Availability of data and materials}

The datasets analyzed during the present study are available from the corresponding author on reasonable request.

\section{Authors' contributions}

FL conceived and designed the study. SD and BG analyzed the data and wrote the manuscript. PW performed statistical analysis and prepared the figures. HH and LL collected the data and critically revised the manuscript for important intellectual content. FL takes responsibility for the honesty and accuracy of the present study. All authors have read and approved the final manuscript.

\section{Ethics approval and consent to participate}

Not applicable.

\section{Patient consent for publication}

Not applicable.

\section{Competing interests}

The authors declare that they have no competing interests.

\section{References}

1. Ferlay J, Soerjomataram I, Dikshit R, Eser S, Mathers C, Rebelo M, Parkin DM, Forman D and Bray F: Cancer incidence and mortality worldwide: Sources, methods and major patterns in GLOBOCAN 2012. Int J Cancer 136: E359-E386, 2015.

2. Bertuccio P, Chatenoud L, Levi F, Praud D, Ferlay J, Negri E, Malvezzi M and La Vecchia C: Recent patterns in gastric cancer: A global overview. Int J Cancer 125: 666-673, 2009.

3. Allemani C, Weir HK, Carreira H, Harewood R, Spika D, Wang XS, Bannon F, Ahn JV, Johnson CJ, Bonaventure A, et al: Global surveillance of cancer survival 1995-2009: Analysis of individual data for $25,676,887$ patients from 279 population-based registries in 67 countries (CONCORD-2). Lancet 385: 977-1010, 2015.

4. Pectasides D, Mylonakis A, Kostopoulou M, Papadopoulou M, Triantafillis D, Varthalitis J, Dimitriades M and Athanassiou A: CEA, CA 19-9 and CA-50 in monitoring gastric carcinoma. Am J Clin Oncol 20: 348-353, 1997.

5. Ara H, Takagishi M, Enomoto A, Asai M, Ushida K, Asai N, Shimoyama Y, Kaibuchi K, Kodera Y and Takahashi M: Role for Daple in non-canonical Wnt signaling during gastric cancer invasion and metastasis. Cancer Sci 107: 133-139, 2016.

6. Yang AP, Liu J, Lei HY, Zhang QW, Zhao L and Yang GH: CA72-4 combined with CEA, CA125 and CA19-9 improves the sensitivity for the early diagnosis of gastric cancer. Clin Chim Acta 437: 183-186, 2014.

7. Yang S and Chung HC: Novel biomarker candidates for gastric cancer. Oncol Rep 19: 675-680, 2008.

8. Sun H: Identification of key genes associated with gastric cancer based on DNA microarray data. Oncol Lett 11: 525-530, 2016.

9. Hu K and Chen F: Identification of significant pathways in gastric cancer based on protein-protein interaction networks and cluster analysis. Genet Mol Biol 35: 701-708, 2012.
10. Zhang MH, Shen QH, Qin ZM, Wang QL and Chen X: Systematic tracking of disrupted modules identifies significant genes and pathways in hepatocellular carcinoma. Oncol Lett 12: 3285-3295, 2016.

11. Gautier L, Cope L, Bolstad BM and Irizarry RA: Affy-analysis of Affymetrix GeneChip data at the probe level. Bioinformatics 20: 307-315, 2004.

12. Benito M, Parker J, Du Q, Wu J, Xiang D, Perou CM and Marron JS: Adjustment of systematic microarray data biases. Bioinformatics 20: 105-114, 2004.

13. Shen ZG, Guo JL and Li DS: Screening of differentially expressed genes related to severe sepsis induced by multiple trauma with DNA microarray. Eur Rev Med Pharmacol Sci 18: 734-739, 2014

14. von Mering C, Huynen M, Jaeggi D, Schmidt S, Bork P and Snel B: STRING: A database of predicted functional associations between proteins. Nucleic Acids Res 31: 258-261, 2003.

15. Shannon P, Markiel A, Ozier O, Baliga NS, Wang JT, Ramage D, Amin N, Schwikowski B and Ideker T: Cytoscape: A software environment for integrated models of biomolecular interaction networks. Genome Res 13: 2498-2504, 2003.

16. Jin Z, Jiang $\mathrm{W}$ and Wang L: Biomarkers for gastric cancer: Progression in early diagnosis and prognosis (Review). Oncol Lett 9: 1502-1508, 2015.

17. Uppal DS and Powell SM: Genetics/genomics/proteomics of gastric adenocarcinoma. Gastroenterol Clin North Am 42: 241-260, 2013.

18. Fan B, Dachrut S, Coral H, Yuen ST, Chu KM, Law S, Zhang L, Ji J, Leung SY and Chen X: Integration of DNA copy number alterations and transcriptional expression analysis in human gastric cancer. PLoS One 7: e29824, 2012.

19. Takeno A, Takemasa I, Doki Y, Yamasaki M, Miyata H, Takiguchi S, Fujiwara Y, Matsubara K and Monden M: Integrative approach for differentially overexpressed genes in gastric cancer by combining large-scale gene expression profiling and network analysis. Br J Cancer 99: 1307-1315, 2008.

20. Bauer L, Langer R, Becker K, Hapfelmeier A, Ott K, Novotny A, Hofler $\mathrm{H}$ and Keller G: Expression profiling of stem cell-related genes in neoadjuvant-treated gastric cancer: A NOTCH2, GSK3B and beta-catenin gene signature predicts survival. PLoS One 7: e44566, 2012.

21. Hudler P: Challenges of deciphering gastric cancer heterogeneity. World J Gastroenterol 21: 10510-10527, 2015.

22. Cancer Genome Atlas Research Network: Comprehensive molecular characterization of gastric adenocarcinoma. Nature 513: 202-209, 2014

23. Zhang XP, Bai ZB, Chen BA, Feng JF, Yan F, Jiang Z, Zhong YJ, Wu JZ, Chen L, Lu ZH et al: Polymorphisms of dihydropyrimidine dehydrogenase gene and clinical outcomes of gastric cancer patients treated with fluorouracil-based adjuvant chemotherapy in Chinese population. Chin Med J (Engl) 125: 741-746, 2012.

24. McGranahan N and Swanton C: Biological and therapeutic impact of intratumor heterogeneity in cancer evolution. Cancer Cell 27: 15-26, 2015.

25. Gerashchenko TS, Denisov EV, Litviakov NV, Zavyalova MV, Vtorushin SV, Tsyganov MM, Perelmuter VM and Cherdyntseva NV: Intratumor heterogeneity: Nature and biological significance. Biochemistry (Mosc) 78: 1201-1215, 2013.

26. Pinheiro Ddo R, Ferreira WA, Barros MB, Araujo MD, Rodrigues-Antunes S and Borges Bdo N: Perspectives on new biomarkers in gastric cancer: Diagnostic and prognostic applications. World J Gastroenterol 20: 11574-11585, 2014.

27. Chang F, Lee JT, Navolanic PM, Steelman LS, Shelton JG, Blalock WL, Franklin RA and Mccubrey JA: Involvement of PI3K/Akt pathway in cell cycle progression, apoptosis and neoplastic transformation: A target for cancer chemotherapy. Leukemia 17: 590-603, 2003.

28. Kikuchi A: Tumor formation by genetic mutations in the components of the Wnt signaling pathway. Cancer Sci 94: 225-229, 2010.

29. Uchi R, Kogo R, Kawahara K, Sudo T, Yokobori T, Eguchi H, Sugimachi K, Maehama T, Mori M, Suzuki A, et al: PICT1 regulates TP53 via RPL11 and is involved in gastric cancer progression. Br J Cancer 109: 2199-2206, 2013.

30. Kim HJ and Oh SC: Novel systemic therapies for advanced gastric cancer. J Gastric Cancer 18: 1-19, 2018.

31. Bang YJ, Van Cutsem E, Feyereislova A, Chung HC, Shen L, Sawaki A, Lordick F, Ohtsu A, Omuro Y, Satoh T, et al: Trastuzumab in combination with chemotherapy versus chemotherapy alone for treatment of HER2-positive advanced gastric or gastro-oesophageal junction cancer (ToGA): A phase 3, open-label, randomised controlled trial. Lancet 376: 687-697, 2010 
32. Rüschoff J, Hanna W, Bilous M, Hofmann M, Osamura RY, Penault-Llorca F, van de Vijver M and Viale G: HER2 testing in gastric cancer: A practical approach. Mod Pathol 25: 637-650, 2012.

33. Storlazzi CT, Wozniak A, Panagopoulos I, Sciot R, Mandahl N, Mertens F and Debiec-Rychter M: Rearrangement of the COL12A1 and COL4A5 genes in subungual exostosis: molecular cytogenetic delineation of the tumor-specific translocation $\mathrm{t}(\mathrm{X} ; 6)(\mathrm{q} 13-14 ; \mathrm{q} 22)$. Int J Cancer 118: 1972-1976, 2006.

34. Januchowski R, Swierczewska M, Sterzyńska K, Wojtowicz K, Nowicki $\mathrm{M}$ and Zabel $\mathrm{M}$ : Increased expression of several collagen genes is associated with drug resistance in ovarian cancer cell lines. J Cancer 7: 1295-1310, 2016.

35. Verghese ET, Drury R, Green CA, Holliday DL, Lu X, Nash C, Speirs V, Thorne JL, Thygesen HH, Zougman A, et al: MiR-26b is down-regulated in carcinoma-associated fibroblasts from ER-positive breast cancers leading to enhanced cell migration and invasion. J Pathol 231: 388-399, 2013.

36. Mikula M, Rubel T, Karczmarski J, Goryca K, Dadlez M and Ostrowski J: Integrating proteomic and transcriptomic high-throughput surveys for search of new biomarkers of colon tumors. Funct Integr Genomics 11: 215-224, 2011.

37. Hayes JD and Strange RC: Glutathione S-transferase polymorphisms and their biological consequences. Pharmacology 61: 154-166, 2000.

38. Hayes JD and Pulford DJ: The glutathione S-transferase supergene family: Regulation of GST and the contribution of the isoenzymes to cancer chemoprotection and drug resistance. Crit Rev Biochem Mol Biol 30: 445-600, 1995.

39. Drozd E, Krzysztoń-Russjan J, Marczewska J, Drozd J, Bubko I, Bielak M, Lubelska K, Wiktorska K, Chilmonczyk Z, Anuszewska E and Gruber-Bzura B: Up-regulation of glutathione-related genes, enzyme activities and transport proteins in human cervical cancer cells treated with doxorubicin. Biomed Pharmacother 83: 397-406, 2016.

40. Wang H, Meyer CA, Fei T, Wang G, Zhang F and Liu XS: A systematic approach identifies FOXA1 as a key factor in the loss of epithelial traits during the epithelial-to-mesenchymal transition in lung cancer. BMC Genomics 14: 680, 2013.

41. Chen C, Zhang LG, Liu J, Han H, Chen N, Yao AL, Kang SS Gao WX, Shen H, Zhang LJ et al: Bioinformatics analysis of differentially expressed proteins in prostate cancer based on proteomics data. Onco Targets Ther 9: 1545-1557, 2016.

42. Fan BL, Zhu WL, Zou GL, Luo GS, Xu CL and Zhao WX: Cloning and identification of fibrinogen gamma polypeptide (FGG) gene differentially expressed in human hepatocellular carcinoma. Ai Zheng 23: 249-253, 2004.

43. Bloomston M, Zhou JX, Rosemurgy AS, Frankel W, Muro-Cacho CA and Yeatman TJ: Fibrinogen gamma overexpression in pancreatic cancer identified by large-scale proteomic analysis of serum samples. Cancer Res 66: 2592-2599, 2006.
44. Tang L, Liu K, Wang J, Wang C, Zhao P and Liu J: High preoperative plasma fibrinogen levels are associated with distant metastases and impaired prognosis after curative resection in patients with colorectal cancer. J Surg Oncol 102: 428-432, 2010

45. Ghezzi F, Cromi A, Siesto G, Giudici S, Serati M, Formenti G and Franchi M: Prognostic significance of preoperative plasma fibrinogen in endometrial cancer. Gynecol Oncol 119: 309-313, 2010.

46. Yano HKitayama J, Hatano K, Tsuno N, Osada T, Watanabe T, Tsuruo T, Muto $\mathrm{T}$ and Nagawa $\mathrm{H}$ : Clustered cancer cells show a distinct adhesion behavior from single cell form under physiological shear conditions. J Exp Clin Cancer Res 20: 407-412, 2001.

47. Nieswandt B, Hafner M, Echtenacher B and Mannel DN: Lysis of tumor cells by natural killer cells in mice is impeded by platelets. Cancer Res 59: 1295-1300, 1999.

48. Palumbo JS, Kombrinck KW, Drew AF, Grimes TS, Kiser JH, Degen JL and Bugge TH: Fibrinogen is an important determinant of the metastatic potential of circulating tumor cells. Blood 96: 3302-3309, 2000.

49. Palumbo JS, Potter JM, Kaplan LS, Talmage K, Jackson DG and Degen JL: Spontaneous hematogenous and lymphatic metastasis, but not primary tumor growth or angiogenesis, is diminished in fibrinogen-deficient mice. Cancer Res 62: 6966-6972, 2002.

50. Yamashita H, Kitayama J, Kanno N, Yatomi Y and Nagawa H: Hyperfibrinogenemia is associated with lymphatic as well as hematogenous metastasis and worse clinical outcome in T2 gastric cancer. BMC Cancer 6: 147, 2006.

51. Davalieva K, Kiprijanovska S, Komina S, Petrusevska G, Zografska NC and Polenakovic M: Proteomics analysis of urine reveals acute phase response proteins as candidate diagnostic biomarkers for prostate cancer. Proteome Sci 13: 2, 2015.

52. Zhu WL, Fan BL, Liu DL and Zhu WX: Abnormal expression of fibrinogen gamma (FGG) and plasma level of fibrinogen in patients with hepatocellular carcinoma. Anticancer Res 29: 2531-2534, 2009.

53. Chan KY, Lai PB, Squire JA, Beheshti B, Wong NL, Sy SM and Wong N: Positional expression profiling indicates candidate genes in deletion hotspots of hepatocellular carcinoma. Mod Pathol 19: 1546-1554, 2006.

54. Son HJ, Park JW, Chang HJ, Kim DY, Kim BC, Kim SY, Park SC, Choi HS and Oh JH: Preoperative plasma hyperfibrinogenemia is predictive of poor prognosis in patients with nonmetastatic colon cancer. Ann Surg Oncol 20: 2908-2913, 2013.

55. Fort A, Borel C, Migliavacca E, Antonarakis SE, Fish RJ and Neerman-Arbez M: Regulation of fibrinogen production by microRNAs. Blood 116: 2608-2615, 2010.

This work is licensed under a Creative Commons Attribution-NonCommercial-NoDerivatives 4.0 International (CC BY-NC-ND 4.0) License. 